\title{
Relapsing Fever Infection Manifesting as Aseptic Meningitis, Texas, USA
}

\author{
Lisa Ellis, Michael W. Curtis, Sarah M. Gunter, Job E. Lopez
}

\section{MedscapeACTIVITY}

In support of improving patient care, this activity has been planned and implemented by Medscape, LLC and Emerging Infectious Diseases. Medscape, LLC is jointly accredited by the Accreditation Council for Continuing Medical Education (ACCME), the Accreditation Council for Pharmacy Education (ACPE), and the American Nurses Credentialing Center (ANCC), to provide continuing education for the healthcare team.

Medscape, LLC designates this Journal-based CME activity for a maximum of 1.00 AMA PRA Category 1 Credit(s) ${ }^{\mathrm{TM}}$ Physicians should claim only the credit commensurate with the extent of their participation in the activity.

Successful completion of this CME activity, which includes participation in the evaluation component, enables the participant to earn up to $1.0 \mathrm{MOC}$ points in the American Board of Internal Medicine's (ABIM) Maintenance of Certification (MOC) program. Participants will earn MOC points equivalent to the amount of CME credits claimed for the activity. It is the CME activity provider's responsibility to submit participant completion information to ACCME for the purpose of granting ABIM MOC credit.

All other clinicians completing this activity will be issued a certificate of participation. To participate in this journal CME activity: (1) review the learning objectives and author disclosures; (2) study the education content; (3) take the post-test with a $75 \%$ minimum passing score and complete the evaluation at http://www.medscape.org/journal/eid; and (4) view/print certificate. For CME questions, see page 2751.

Release date: September 20, 2021; Expiration date: September 20, 2022

\section{Learning Objectives}

Upon completion of this activity, participants will be able to:

- Describe clinical presentation and course in a case of neuroborreliosis in Austin, Texas, that was initially thought to be Lyme disease (LD)

- Determine retrospective serological analysis and other laboratory findings in a case of neuroborreliosis in Austin, Texas, that was initially thought to be LD

- Identify clinical and public health implications of findings in this case of neuroborreliosis in Austin, Texas, that was initially thought to be LD.

\section{CME Editor}

Terie A. Grant, BS, Copyeditor, Emerging Infectious Diseases. Disclosure: Terie A. Grant, BS, has disclosed no relevant financial relationships.

\section{CME Author}

Laurie Barclay, MD, freelance writer and reviewer, Medscape, LLC. Disclosure: Laurie Barclay, MD, has disclosed no relevant financial relationships.

\section{Authors}

Disclosures: Lisa C. Ellis, MD; Michael W. Curtis, PhD; Sarah M. Gunter, PhD, MPH; and Job E. Lopez, PhD, have disclosed no relevant financial relationships.

Author affiliations: Austin Infectious Disease Consultants, Austin, Texas, USA (L. Ellis); Baylor College of Medicine, Houston, Texas (M.W. Curtis, S.M. Gunter, J.E. Lopez) 
Tickborne relapsing fever spirochetes are an overlooked cause of disease around the globe. We report a case of tickborne relapsing fever in a patient in Texas, USA, who had a single febrile episode and gastrointestinal and neurologic symptoms. Immunoblot analysis using recombinant Borrelia immunogenic protein A implicated Borrelia turicatae as the causative agent.

$\mathrm{T}$ ickborne relapsing fever (TBRF) spirochetes are globally neglected pathogens. Borrelia turicatae is found in the southwestern and eastern United States into Latin America (1), and high-risk populations include military personnel, outdoor enthusiasts, and impoverished undocumented immigrants (2-4). However, evidence indicates the presence of endemic foci of $B$. turicatae in metropolitan cities of Texas, USA $(4,5)$.

TBRF is often misdiagnosed because of the nonspecific manifestations of the disease. More than $90 \%$ of patients experience recurrent febrile episodes, rigors, headache, and myalgia (6). Previous work suggests that $B$. turicatae is similar to Old World species, manifesting with neurologic complications (7).
However, these diagnoses were made on the basis of a priori assumptions, and the causative agents were never confirmed.

We report a case of neuroborreliosis in Austin, Texas, USA, that was initially suspected to be Lyme disease (LD). A retrospective serologic analysis was performed using the diagnostic antigen, Borrelia immunogenic protein A (BipA). This antigen is absent from LD-causing spirochetes and might be a species-specific antigen for North American TBRF Borrelia $(8,9)$.

\section{The Study}

The patient was a previously healthy 30-year-old man residing in Austin near a creek greenbelt that he frequented (Figure 1); he had no recent travel outside the city. On March 5, 2020, he experienced acute dizziness, headache, myalgia, vomiting, chills, and fever of $37.8^{\circ} \mathrm{C}$ (reference $36.1^{\circ} \mathrm{C}-37.2^{\circ} \mathrm{C}$ ). Symptoms were attributed to a foodborne illness, and he improved after several days. However, he continued to experience dizziness, headache, fatigue, myalgia, and intermittent severe night sweats, with no report of further fever.

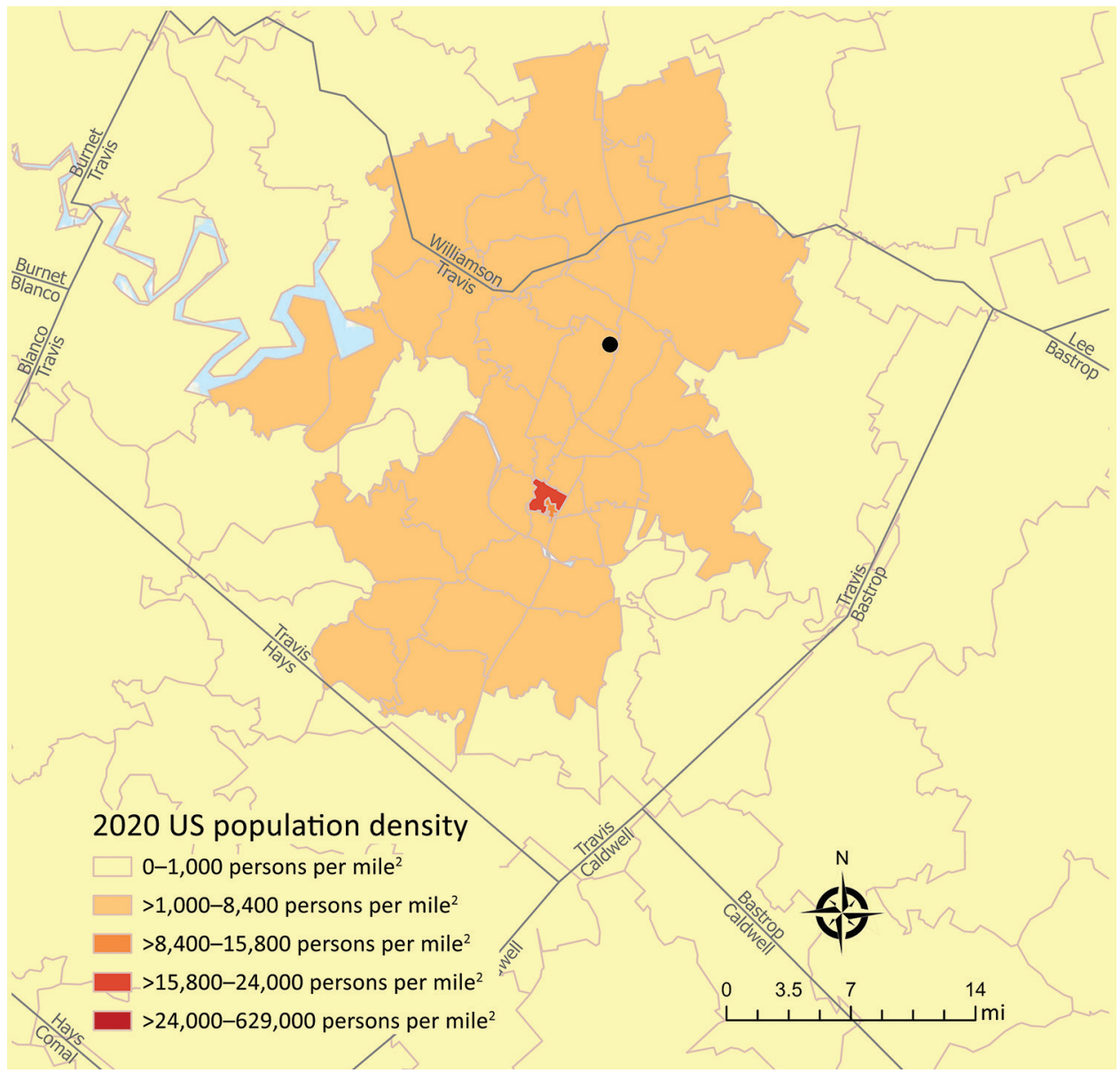

Figure 1. Suspected Borrelia exposure site within city limits for a patient in Austin, Texas, USA. The patient's suspected exposure location (black circle, Walnut Creek Metropolitan Park) was overlayed on a population density by ZIP code map. County boundaries are displayed as gray lines. Population density data was sourced from Esri's U.S. Updated Demographic (2020/2025) Data (https://www.esri.com). 
Within 2 weeks, he had Bell's palsy on his left side, and the primary care physician ordered a blood analysis. Results for complete blood count, electrolytes, blood urea nitrogen, creatinine, and liver enzymes were unremarkable. Erythrocyte sedimentation rate was $62 \mathrm{~mm} / \mathrm{h}$ (reference $<15 \mathrm{~mm} / \mathrm{h}$ ), and C-reactive protein was $97.5 \mathrm{mg} / \mathrm{L}$ (reference $<8.0 \mathrm{mg} / \mathrm{L}$ ). Valacyclovir (1 g orally $3 \times / \mathrm{d}$ for $7 \mathrm{~d})$ and prednisone $(20$ mg orally $2 \times / d$ for $5 \mathrm{~d}$ ) were prescribed, with partial improvement. Subsequent blood testing showed the erythrocyte sedimentation rate declined to $41 \mathrm{~mm} / \mathrm{h}$, and C-reactive protein declined to $43.2 \mathrm{mg} / \mathrm{L}$.

Two weeks later, he had Bell's palsy on his right side, blurred vision, tinnitus, and cervical lymph node enlargement. Dizziness, headache, and fatigue continued. Results of complete blood count and metabolic panel were unremarkable. The patient underwent magnetic resonance imaging of the brain with contrast, which revealed faint nonspecific enhancement in the right internal auditory canal. High-resolution imaging of the 7th and 8th cranial nerves was not performed.

The patient's wife reported removing ticks from herself and a pet 4 weeks before the patient's illness began, and LD was suspected. A 2-tiered antibody test was performed. The enzyme immunoassay result was 2.43 (>1.09 considered positive). The LD IgM immunoblot was positive for the $23 \mathrm{kDa}$ and $39 \mathrm{kDa}$ bands, but the IgG immunoblot was negative.

The patient was referred to an infectious disease specialist who suspected TBRF and ordered a lumbar puncture. Clear, colorless cerebrospinal fluid (CSF) was recovered, and the analysis revealed 124 leukocytes/UL (reference <5 leukocytes/UL) with 85\% lymphocytes, $10 \%$ monocytes, $5 \%$ large mononuclear cells, and 0\% erythrocytes/UL. CSF protein was 103 $\mathrm{mg} / \mathrm{dL}$ (reference $15.0-45.0 \mathrm{mg} / \mathrm{dL}$ ), and glucose was $52 \mathrm{mg} / \mathrm{dL}$ (reference $40-70 \mathrm{mg} / \mathrm{dL}$ ). CSF was analyzed by the Associated Regional and University Pathologists laboratory for LD and TBRF spirochete DNA, the Venereal Disease Research Laboratory test for neurosyphilis, and the Biofire Filmarray Meningitis/Encephalitis (bioMérieux, https://www.biomerieux-usa.com) panel that detects 6 bacterial and 7 viral pathogens. All test results were negative.

Given the patient's clinical history, intravenous ceftriaxone was administered $(2 \mathrm{~g} / \mathrm{d}$ for $14 \mathrm{~d})$, and he showed considerable improvement within 4 days of treatment. Upon completion of antibiotics, all symptoms were resolved except for minimal lower right facial weakness. Deidentified serum samples and CSF collected 5 weeks after the onset of illness were sent to Baylor College of Medicine (Houston, TX, USA) for additional testing.
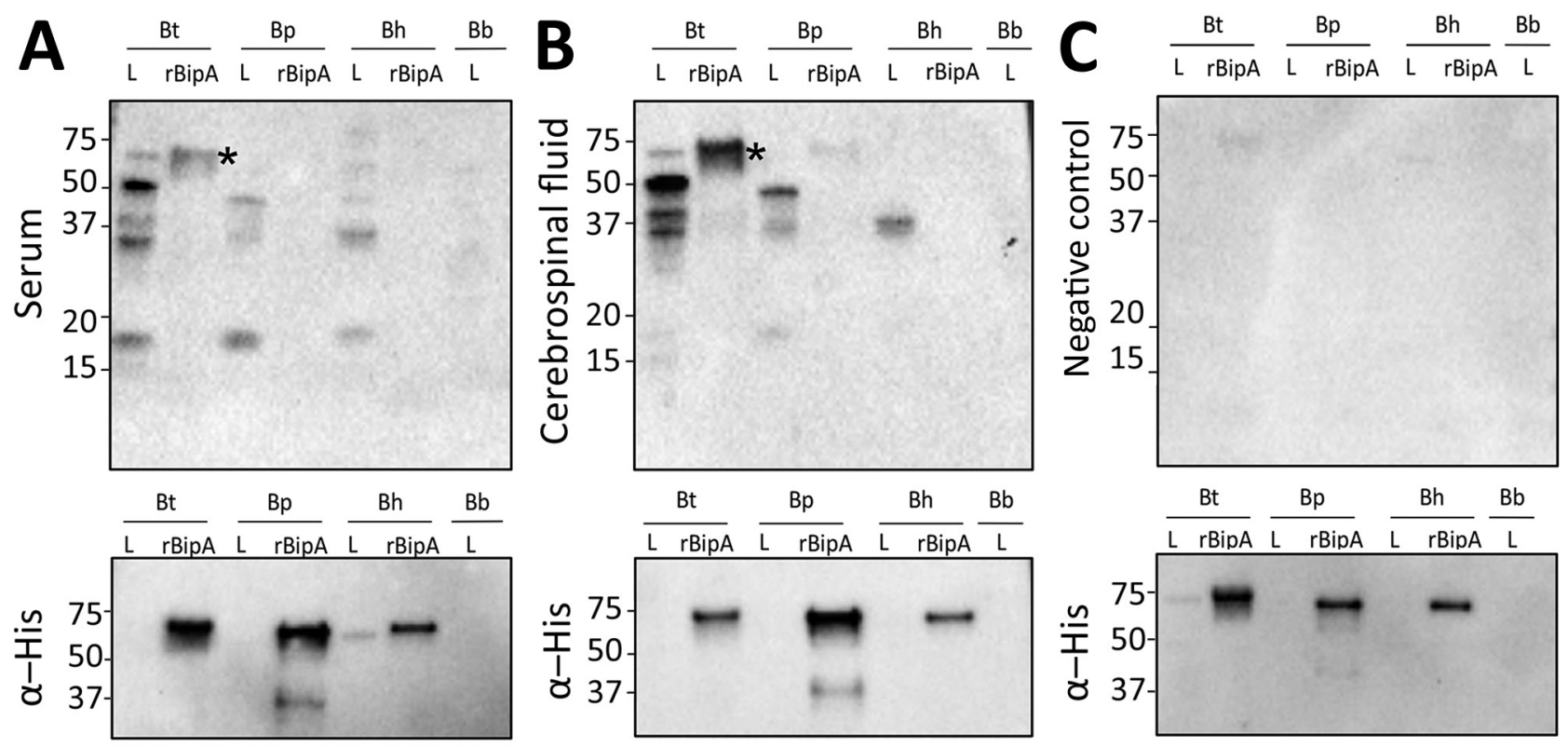

Figure 2. Immunoblots assessing antibody responses to Borrelia protein lysates and rBipA in samples from a patient in Texas, USA, and a control sample. A, B) Serum (A, upper panel) and cerebrospinal fluid (B, upper panel) samples were used to detect reactivity to Borrelia protein lysates and to rBipA from each species of tickborne relapsing fever spirochete. C) Negative human serum sample (upper panel) and immunoblots (bottom panel) that were reprobed with a monoclonal antibody for the histidine residues fused on the $\mathrm{N}$ terminus of each recombinant protein. Asterisks $\left(^{*}\right)$ indicates $\mathrm{rBipA}$, which is $\approx 65 \mathrm{kDa}$. Molecular masses in $\mathrm{kDa}$ are indicated on the left of each immunoblot. Bb, Borreliela (Borrelia) burgdorferi; Bh, B. hermsii; BipA, Borrelia immunogenic protein A; Bp, B. parkeri; Bt,

B. turicatae; rBipA, recombinant BipA. 
No spirochetes were recovered from the CSF nor was DNA detected; therefore, we performed serologic tests using recombinant $\mathrm{BipA}(\mathrm{rBipA})(8,9)$. We generated expression constructs for B. turicatae, B. parkeri, and B. hermsii rBipA by using GenScript (GenScript, https://www.genscript.com) in the pET19b vector. We purified recombinant proteins and performed immunoblotting and ELISA, as previously described (8). For immunoblots, we used protein lysates from $B$. turicatae 91E135, B. hermsii DAH, and B. parkeri SLO. We probed immunoblots with the patient's serum sample and CSF diluted 1:200. Only the serum sample was diluted 2-fold from 1:200 to 1:256,000 for the ELISA because the CSF was depleted in prior assays. The secondary antibody was goat anti-human IgA, IgG, and IgM (Millipore, https://www.emdmillipore. com). We repeated serologic assays twice.

Serologic assays indicated likely exposure to $B$. turicatae. Strong responses were detected with the serum sample and CSF to $B$. turicatae protein lysates and rBipA (Figure 2, panels A and B upper). Antibodies in the patient's serum and CSF cross-reacted with protein lysates from $B$. parkeri and B. hermsii, but reactivity to rBipA from these species was undetectable (Figure 2, panels A, B, top images). In addition, reactivity to $B$. burgdorferi protein lysates was undetectable (Figure 2, panels A, B, top images). A negative control serum sample from a subject without history of TBRF failed to detect proteins (Figure 2, panel C, top image). Reprobing immunoblots with a monoclonal antibody for the histidine residues fused to rBipA demonstrated that protein was electrophoresed and transferred to membranes (Figure 2, bottom images). ELISA further indicated infection attributable to $B$. turicatae with antibody titers to $B$. turicatae rBipA between 1:400 to 1:800, and responses to $B$. parkeri and $B$. hermsii rBipA were undetectable.

\section{Conclusions}

This study reports a case of neurologic TBRF likely caused by B. turicatae. The hallmark of TBRF is recurrent febrile episodes (6), but this patient had a single febrile episode, nausea, and predominantly neurologic symptoms. B. turicatae has been suspected to cause neurologic symptoms including facial paralysis, vertigo, hearing loss, delirium, and hallucinations (10). However, past diagnoses were attributed solely on the basis of the geographic range of the probable pathogen and were not empirically confirmed.

This study demonstrated that rBipA could aid in identifying the TBRF species causing infection. It was unlikely that the patient was exposed to B. hermsii and $B$. parkeri because of his travel history, but we used this opportunity to assess serologic crossreactivity to rBipA from these 2 species. Similar to prior work with B. turicatae-infected laboratory animals (8), we detected no crossreactive patient antibodies to $B$. hermsii rBipA. This finding was expected given that the proteins share $\approx 35 \%$ amino acid identity (11). Of note, rBipA could differentiate between infections caused by B. parkeri and B. turicatae, which share $\approx 75 \%$ amino acid identity (11).

In summary, B. turicatae is often misdiagnosed, and healthcare providers should understand the pathogen's circulation $(2,5,11)$. Endemic foci have been identified in Florida, USA, and within and around the 4 largest cities of Texas (Austin, San Antonio, Dallas, and Houston) (1-3,5,7). With urban expansion and the incorporation of greenbelts into metropolitan areas, $B$. turicatae should be considered in cases of fever with neurologic symptoms when the Lyme antibody test is positive but prevalence of LD is not epidemiologically supported.

\section{Acknowledgments}

We thank Aparna Krishnavajhala and Alexander Kneubehl for critical review of this manuscript and Tom Schwan for originally providing B. hermsii, B. turicatae, and B. parkeri isolates for serology.

This work was supported by funding from National Institutes of Health grant no. AI144187 (JEL). Michael W. Curtis was supported through the Infection and Immunity T32 Fellowship at Baylor College of Medicine (grant no. T32AI055413).

\section{About the Author}

Dr. Ellis is an infectious disease specialist in Austin, Texas, USA, in clinical practice with Austin Infectious Disease Consultants. Her research interests include HIV and atypical mycobacterium infections.

\section{References}

1. Lopez JE, Krishnavahjala A, Garcia MN, Bermudez S. Tick-borne relapsing fever spirochetes in the Americas. Vet Sci. 2016;3:1-18. https://doi.org/10.3390/vetsci3030016

2. Rawlings JA. An overview of tick-borne relapsing fever with emphasis on outbreaks in Texas. Tex Med. 1995;91:56-9.

3. Christensen AM, Pietralczyk E, Lopez JE, Brooks C, Schriefer ME, Wozniak E, et al. Diagnosis and management of Borrelia turicatae infection in febrile soldier, Texas, USA. Emerg Infect Dis. 2017;23:883-4. https://doi.org/10.3201/ eid2305.162069

4. Campbell SB, Klioueva A, Taylor J, Nelson C, Tomasi S, Replogle A, et al. Evaluating the risk of tick-borne relapsing fever among occupational cavers - Austin, TX, 2017. Zoonoses Public Health. 2019;66:579-86. https://doi.org/ $10.1111 /$ zph.12588 
5. Bissett JD, Ledet S, Krishnavajhala A, Armstrong BA, Klioueva A, Sexton C, et al. Detection of tickborne relapsing fever spirochete, Austin, Texas, USA. Emerg Infect Dis. 2018;24:2003-9. https://doi.org/10.3201/eid2411.172033

6. Dworkin MS, Schwan TG, Anderson DE Jr, Borchardt SM. Tick-borne relapsing fever. Infect Dis Clin North Am. 2008;22:449-68, viii. https:/ / doi.org/10.1016/ j.idc. 2008.03 .006

7. Gillespie JO. Relapsing fever in the United States. JAMA. 1935;104:1878-81. https://doi.org/10.1001/ jama.1935.02760210010003

8. Lopez JE, Wilder HK, Boyle W, Drumheller LB, Thornton JA, Willeford B, et al. Sequence analysis and serological responses against Borrelia turicatae BipA, a putative species-specific antigen. PLoS Negl Trop Dis. 2013;7:e2454. https:// doi.org/10.1371/journal.pntd.0002454

9. Lopez JE, Schrumpf ME, Nagarajan V, Raffel SJ, McCoy BN, Schwan TG. A novel surface antigen of relapsing fever spirochetes can discriminate between relapsing fever and Lyme borreliosis. Clin Vaccine Immunol. 2010;17:564-71. https://doi.org/10.1128/CVI.00518-09

10. Cadavid D, Barbour AG. Neuroborreliosis during relapsing fever: review of the clinical manifestations, pathology, and treatment of infections in humans and experimental animals. Clin Infect Dis. 1998;26:151-64. https://doi.org/10.1086/516276

11. Wilder HK, Wozniak E, Huddleston E, Tata SR, Fitzkee NC, Lopez JE. Case report: a retrospective serological analysis indicating human exposure to tick-borne relapsing fever spirochetes in Texas. PLoS Negl Trop Dis. 2015;9:e0003617. https://doi.org/10.1371/journal.pntd.0003617

Address for correspondence: Job Lopez, Baylor College of Medicine, One Baylor Plaza, Houston, TX 77030, USA; email: job.lopez@bcm.edu

\section{The Public Health Image Library}
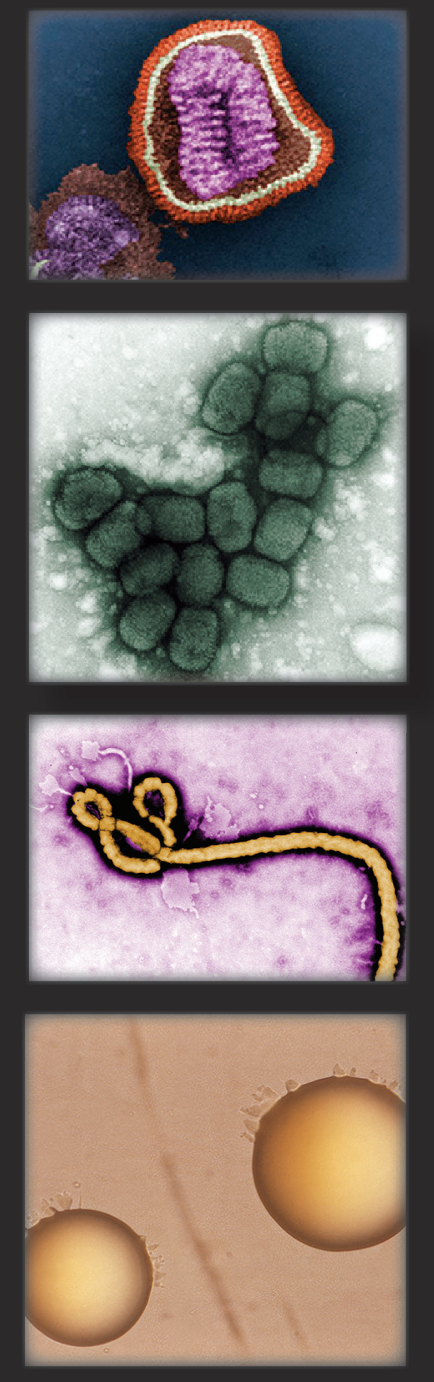
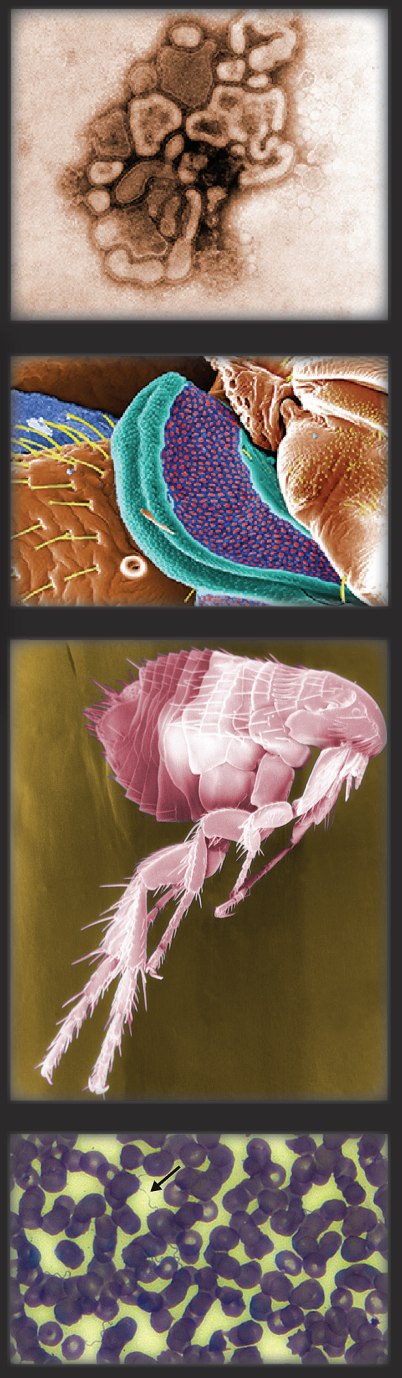

The Public Health Image Library (PHIL), Centers for Disease Control and Prevention, contains thousands of public healthrelated images, including high-resolution (print quality) photographs, illustrations, and videos.

PHIL collections illustrate current events and articles, supply visual content for health promotion brochures, document the effects of disease, and enhance instructional media.

PHIL images, accessible to PC and Macintosh users, are in the public domain and available without charge.

Visit PHIL at: http: / / phil.cdc.gov/phil 